\title{
The 3D Arm Modeling and Position Control
}

\author{
Wei-fang Wang ${ }^{* 1}$, Pao-Hwa Yang ${ }^{2}$, Shiu-Ping Wang ${ }^{2}$ and Lan $\mathrm{Xu}^{1}$ \\ ${ }^{1}$ Suzhou Vocational Institute of Industrial Technology, Suzhou, Jiangsu 215104, China \\ ${ }^{2}$ Hsiuping University of Science \& Technology, No.11 Gungye Rd., Dali,Taichung,Taiwan,R.O.C \\ ${ }^{*}$ Corresponding author
}

Keywords: Nonlinear, Parameter-dependent, Position Control, Simmechanics.

\begin{abstract}
We employ two approaches to model a 3D arm. In nonlinear, a parameter-dependent structure has been derived. Due to an existing inverse inertia term in the motion equation, a proof of nonsingular inertia is given. In linear, the Simmechanic Visual plot is obtained. By implementing a position control law, both of them can reach the same desired position.
\end{abstract}

\section{Introduction}

In this paper, a study of the constructing a model for a 3 degree of freedom (3D) arm is proposed. In which, our discussion is divided into two parts: one is to derive the equation of motion for 3D arm considered as a multi-body system. Another is to employ Solidwork/Simulink software to obtain a joint-mass plot which input force and sensor can be added for simulation. Although, the described approaches for modeling are different, they can be complementary to each other. As results, the equation motion of 3D arm is a parameter dependent and nonlinear system, which is complex and difficult to solve. As for the system from joint-mass plot is linear, which is intuitively and easily to implement to engineering application.

The $3 \mathrm{D}$ arm has been wildly employed in the autonomous manufacture line. It is constituted of three long arms linked with joints along each end and each joint represents one degree of freedom. With this structure simple, flexible and controllable, it had been investigated by many researchers [1-6]. By utilizing all this developing techniques, the 3D arm becomes more sophisticated and reliable tools which had replaced some human tasks in a plant.

Although there are some achievements in application of 3D arms have been made, a complete discussion of a nonlinear 3D arm model structure in literature is still rare [7-8]. The most reason is the complexity of constructing the equation of motion for a 3 degree of freedom arm. However, in order to fully understand the geometry limitation of 3D arm's structure, it is still necessary to investigate the system from nonlinear model.

The paper is organized as the following. In section 2, a detailed structure of 3D arm nonlinear model is presented. In section 3, a position control law is proposed with numerical simulation results. Section 4, the CAD/SIMMECHANIC plot is given. The same control law is implemented in simulation to validate the results. Our conclusion is presented in section 5.

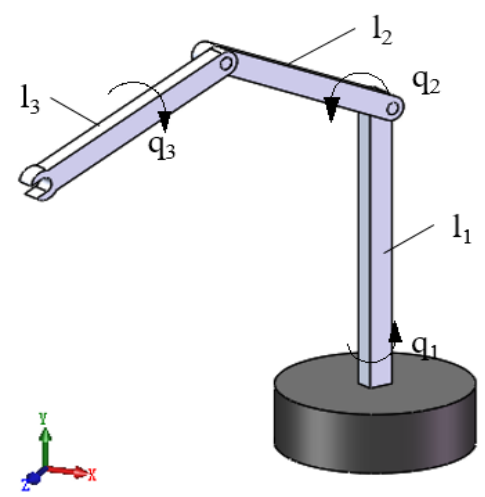

Figure.1 Picture of the 3D arms 


\section{Nonlinear Modeling}

In our 3D arms modeling, the joints which are ${ }^{J_{1}},{ }^{J_{2}},{ }^{J_{3}}$ connected from base to the end of the arm with the length $\left(l_{1}, l_{2}, l_{3}\right)$ and mass $\left(m_{1}, m_{2}, m_{3}\right)$. Each arm with moment inertia $\left(I_{1 x}, I_{1 y}, I_{1 z}\right)$, $\left(I_{2 x}, I_{2 y}, I_{2 z}\right)$ and $\left(I_{3 x}, I_{3 y}, I_{3 z}\right)$. The base joint $J_{1}$ is rotated with ${ }^{y}$-axis. And Joint 2, Joint 3 rotated with $Z$-axis referred to the coordinate system $(x-y-z)$. The joint angles and velocities are defined as $q\left(q_{1}, q_{2}, q_{3}\right)$ and $\dot{q}\left(\dot{q}_{1}, \dot{q}_{2}, \dot{q}_{3}\right)$ as depicted in the figure 1 . The equation of motion can be derived as the following [7-8]:

$$
M_{c}(q) \ddot{q}+C_{c}(q, \dot{q}) \dot{q}+g(q)=\tau
$$

Where $M_{c}(q)$ is a $3 \times 3$ manipulator inertia matrix, $C_{c}(q, \dot{q})$ is a $3 \times 3$ Centripetal and Coriolis matrix, $g(q)$ is $3 \times 1$ vector of gravitational torques, and $\tau$ is $3 \times 1$ control input vector.

$$
\begin{aligned}
& M_{c}=\left[\begin{array}{lll}
M_{11} & M_{12} & M_{13} \\
M_{21} & M_{22} & M_{23} \\
M_{31} & M_{32} & M_{33}
\end{array}\right] \\
& M_{11}=a_{1}+\frac{a_{2}}{8} \cos 2 q_{2}+\frac{a_{3}}{8} \cos \left(2 q_{2}+2 q_{3}\right)+a_{4} \cos q_{2}+\frac{a_{5}}{2}\left(\cos q_{3}+\cos \left(2 q_{2}+q_{3}\right)\right) \\
& +a_{6} \cos \left(q_{2}+q_{3}\right) \\
& M_{12}=M_{13}=M_{21}=M_{31}=0, M_{22}=b_{1}+a_{5} \cos q 3, M_{23}=M_{32}=b_{2}+\frac{a_{5}}{2} \cos q_{3}, M_{33}=b_{2} \\
& C_{c}=\left[\begin{array}{lll}
C_{11} & C_{12} & C_{13} \\
C_{21} & C_{22} & C_{23} \\
C_{31} & C_{32} & C_{33}
\end{array}\right] \\
& C_{11}=C_{22}=0 \\
& C_{12}=-\frac{a_{3}}{4} \dot{q}_{1} \sin \left(2 q_{2}+2 q_{3}\right)-\dot{q}_{1}\left(\frac{a_{2}}{4} \sin 2 q_{2}+a_{3} \sin q_{2}+a_{6} \sin \left(q_{2}+q_{3}\right)+a_{5} \sin \left(2 q_{2}+q_{3}\right)\right) \\
& C_{21}=\frac{a_{3}}{8} \dot{q}_{1} \sin \left(2 q_{2}+2 q_{3}\right)+\dot{q}_{1}\left(\frac{a_{2}}{8} \sin 2 q_{2}+\frac{a_{3}}{2} \sin q_{2}+\frac{a_{6}}{2} \sin \left(q_{2}+q_{3}\right)+\frac{a_{5}}{2} \sin \left(2 q_{2}+q_{3}\right)\right) \\
& C_{23}=-\frac{a_{5}}{2}\left(2 \dot{q}_{2}+\dot{q}_{3}\right) \sin q_{3}
\end{aligned}
$$




$$
\begin{gathered}
C_{31}=\frac{1}{4} \dot{q}_{1}\left(2 a_{6}+2 a_{5} \cos q_{2}+a_{3} \cos \left(q_{2}+q_{3}\right)\right) \sin \left(q_{2}+q_{3}\right) \\
C_{32}=\frac{1}{4} a_{5}\left(2 \dot{q}_{5}+\dot{q}_{3}\right) \sin q_{3} \\
C_{33}=-\frac{a_{5}}{4} \dot{q}_{2} \sin q_{3} \\
g_{c}(q)=\left[\begin{array}{c}
g_{1} \\
g_{2} \\
g_{3}
\end{array}\right] \begin{array}{c}
g_{3}=-\frac{1}{2} g d_{3} \sin q_{1} \sin \left(q_{2}+q_{3}\right) \\
g_{1}=-\frac{1}{2} g \cos q_{1}\left(d_{1}+d_{2} \cos q_{2}+d_{3} \cos \left(q_{2}+q_{3}\right)\right) \\
g_{2}=\frac{1}{2} g \sin q_{1}\left(d_{2} \sin q_{2}+d_{3} \sin \left(q_{2}+q_{3}\right)\right)
\end{array}
\end{gathered}
$$

$g$ is the gravity.

$$
\tau=\left[\begin{array}{l}
\tau_{1} \\
\tau_{2} \\
\tau_{3}
\end{array}\right]
$$

The input torque $\tau=\left[\begin{array}{lll}\tau_{1} & \tau_{2} & \tau_{3}\end{array}\right]^{T}$ acts on each joint respectively.

In equations (1)-(5), all the terms $\left(a_{1} \ldots a_{6}, b_{1} \ldots b_{2}, d_{1} \ldots d_{3}\right)$ are related to the system parameter which is defined in the table1.

From (1)-(5), a system matrix can be described as the following:

$$
\left[\begin{array}{c}
\dot{q} \\
\ddot{q}
\end{array}\right]=\left[\begin{array}{cc}
0_{3 \times 3} & I_{3 \times 3} \\
0_{3 \times 3} & -M_{c}^{-1}
\end{array}\right]\left[\begin{array}{c}
q \\
\dot{q}
\end{array}\right]+\left[\begin{array}{c}
0_{3 \times 3} \\
-M_{c}^{-1} g_{c}
\end{array}\right]+\left[\begin{array}{c}
0_{3 \times 3} \\
M_{c}^{-1}
\end{array}\right] \tau
$$

$I$ is the identity matrix.

However, there is an inverse inertia term $M_{c}$ in (6). It is important to verify if $M_{c}$ is nonsingular. It is shown that the inertia is positive definite and symmetry in the following theorem.

Table 1. the system parameter

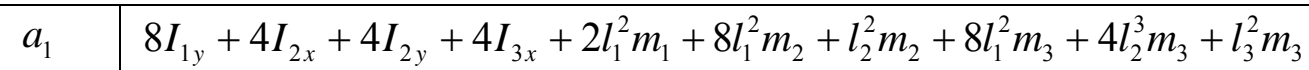




\begin{tabular}{|l|l|}
\hline$a_{2}$ & $-4 I_{2 x}+4 I_{2 y}+l_{2}^{2}\left(m_{2}+4 m_{3}\right)$ \\
\hline$a_{3}$ & $-4 I_{3 x}+4 I_{3 y}+l_{3}^{2} m_{3}$ \\
\hline$a_{4}$ & $l_{1} l_{2}\left(m_{2}+2 m_{3}\right)$ \\
\hline$a_{5}$ & $l_{2} l_{3} m_{3}$ \\
\hline$a_{6}$ & $l_{1} l_{3} m_{3}$ \\
\hline$b_{1}$ & $I_{2 z}+I_{3 z}+\frac{l_{2}^{2} m_{2}}{4}+l_{2}^{2} m_{3}+\frac{l_{3}^{2} m_{3}}{4}$ \\
\hline$b_{2}$ & $I_{3 z}+\frac{l_{3}^{2} m_{3}}{4}$ \\
\hline$d_{1}$ & $l_{1}\left(m_{1}+2\left(m_{2}+m_{3}\right)\right)$ \\
\hline$d_{2}$ & $l_{2}\left(m_{2}+2 m_{3}\right)$ \\
\hline$d_{3}$ & $l_{3} m_{3}$ \\
\hline
\end{tabular}

Theorem 1: there exists a non-trivial solution in (6), if $M_{c}$ is non-singular.

Proof: from (2), $M_{c}=\left[\begin{array}{ccc}M_{11} & 0 & 0 \\ 0 & M_{22} & M_{23} \\ 0 & M_{32} & M_{33}\end{array}\right]$, it is required that $\left|M_{c}\right| \neq 0$

$$
\begin{gathered}
\left|M_{c}\right|=\frac{1}{32}\left(4\left(b_{1}-b_{2}\right) b_{2}-a_{5}^{2} \cos ^{2} q_{3}\right)\left(a_{1}+8 a_{4} \cos q_{2}+a_{2} \cos 2 q_{2}+4 a_{5} \cos q_{3}\right. \\
\left.+8 a_{6} \cos \left(q_{2}+q_{3}\right)+a_{3} \cos \left(2\left(q_{2}+q_{3}\right)\right)+4 a_{5} \cos \left(2 q_{2}+q_{3}\right)\right) \neq 0 \\
\Rightarrow 4\left(b_{1}-b_{2}\right) b_{2}-a_{5}^{2} \cos ^{2} q_{3} \neq 0 \\
\Rightarrow\left(I_{2 z}+I_{3 x}+\frac{l_{2}^{2} m_{2}}{4}+l_{2} m_{3}\right)\left(I_{3 z}+\frac{l_{3}^{2} m_{3}}{4}\right) \neq\left(l_{2} l_{3} m_{3} \cos q_{3}\right)^{2}
\end{gathered}
$$

After arrangement, $\Rightarrow 2 \sqrt{\left(\frac{I_{2 z}}{l_{2}^{2} m_{3}}+\frac{m_{2}}{4 m_{3}}+1\right)\left(\frac{I_{3 z}}{l_{3}^{2} m_{3}}+\frac{1}{4}\right)} \neq \cos q_{3}$

and the term $\left|\frac{I_{2 z}}{I_{2}^{2} m_{3}}+\frac{m_{2}}{4 m_{3}}+1\right|>1,\left|\frac{I_{3 z}}{l_{3}^{2} m_{3}}+\frac{1}{4}\right|>\frac{1}{4}$

so, $2 \sqrt{\left(\frac{I_{2 z}}{l_{2}^{2} m_{3}}+\frac{m_{2}}{4 m_{3}}+1\right)\left(\frac{I_{3 z}}{l_{3}^{2} m_{3}}+\frac{1}{4}\right)}>1$, the inertia matrix $M_{c}$ is symmetry and positive definite.

\section{Position Control}

In this section, a position control law is proposed as described in the next theorem 2.

Theorem 2 (position control): if $q_{d}$ is the desired position, the arm system in (1) can track to it provided the control law $\tau=-K_{p} \widetilde{q}-K_{d} \dot{q} .\left(\widetilde{q}=q-q_{d}, K_{p}\right.$ and $K_{d}$ are adequately chosen constant).[7] 
Proof: the proof is made in the point of view of energy conservation. The occurred kinematic energy of arm $T=\frac{1}{2} \dot{q}^{T} M_{c} \dot{q}$ is provided by the actuator input $\tau$ to drive the arm to some position $q$.

$$
T=\frac{1}{2} \dot{q}^{T} M_{c} \dot{q}=\tau \cdot q
$$

$$
\Rightarrow \frac{1}{2} \frac{d}{d t}\left(\dot{q}^{T} M_{c} \dot{q}\right)=\dot{q}^{T} \tau
$$

and the Lyapunov function $V=\frac{1}{2}\left(\dot{q}^{T} M_{c} \dot{q}+\widetilde{q}^{T} K_{p} \widetilde{q}\right) \Rightarrow \dot{V}=\dot{q}^{T} \tau+\dot{\vec{q}}^{T} K_{p} \widetilde{q}$

however, $\dot{\widetilde{q}}=\dot{q}$ so $\dot{V}=\dot{q}^{T}\left(\tau+K_{p} \widetilde{q}\right)$ plugging into control law then $\dot{V}=-\dot{q}^{T} K_{D} \dot{q} \leq 0$. The system can be stabilized by proposed control law in which means the system can reach to the desired position.

In order to elaborate the proposed position control law, a numerical simulation of the system is given in the next example.

Example 1: the 3D arm physical property is defined in the table 2 and the initial condition $x_{0}=\left[\begin{array}{llllll}0 & 0 & 0 & 1 & 1 & 1\end{array}\right]_{\text {with }}^{T} g_{c}=9.8$ and $q_{d}=\left[\begin{array}{lll}120 & 45 & 60\end{array}\right]$.

Table 2. the arm physical property

\begin{tabular}{|l|l|}
\hline $\operatorname{arm} 1$ & $l_{1}=5, m_{1}=10, I_{1}=\left[\begin{array}{lll}4 & 4 & 2\end{array}\right]$ \\
\hline $\operatorname{arm} 2$ & $l_{2}=4, m_{2}=8, I_{2}=\left[\begin{array}{lll}4 & 4 & 2\end{array}\right]$ \\
\hline $\operatorname{arm} 3$ & $l_{3}=4, m_{3}=8, I_{3}=\left[\begin{array}{lll}2 & 4 & 2\end{array}\right]$ \\
\hline
\end{tabular}

With the help of s-function in Matlab [9-10], the simulation of nonlinear system in (6) can be done and $K_{p}=-100, K_{d}=-10$ are the control gains in figure 2 . The simulation of reaching the desired position is in figure 3 .

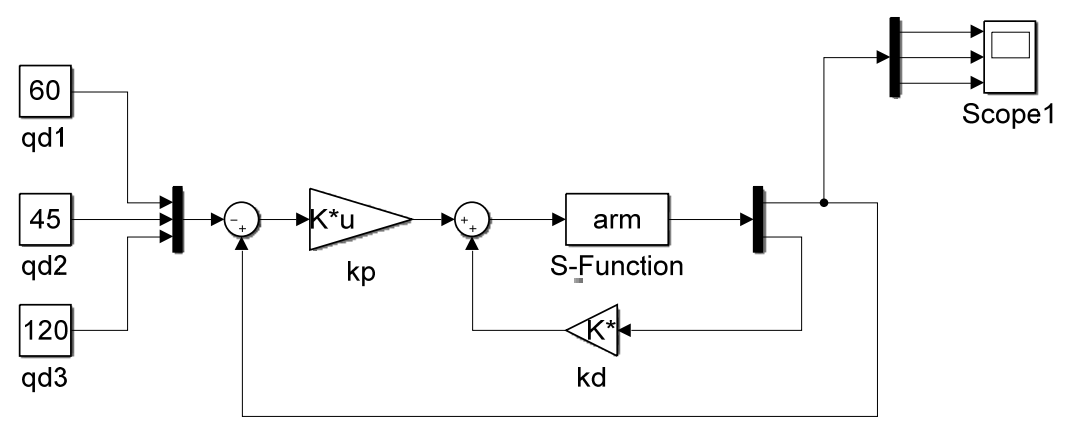

Figure. 2 the nonlinear system 

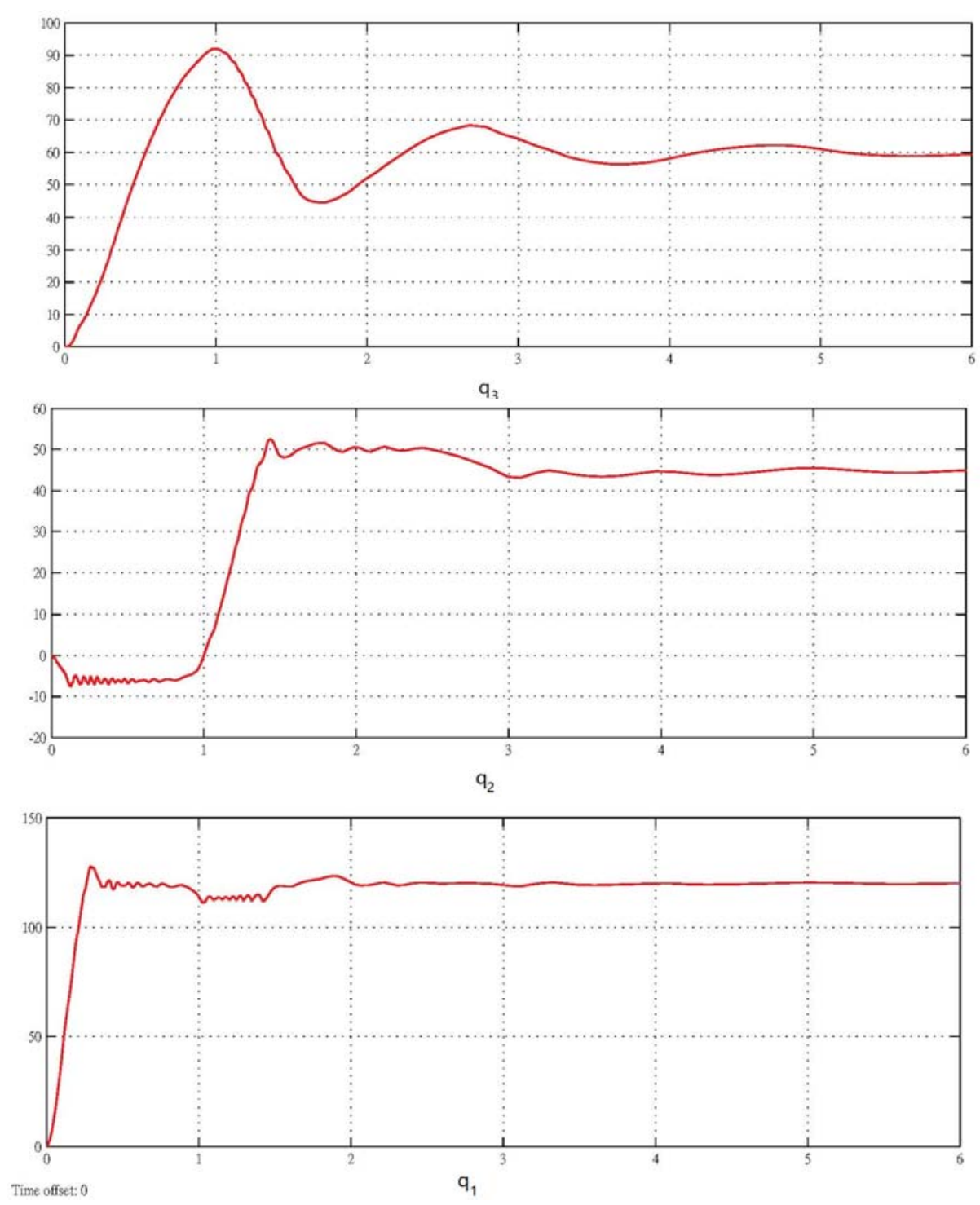

Figure.3 the desired position

\section{CAD/Matlab Simmechanics}

It is quitely intuitive to build a model from 3D visualization CAD software [9-10]. We start from 3D arm SOLIDWOKS engineering drawings. By choosing cast iron as base and aluminum material as three rods, the phsical property of the arm has been determined. Then utilizing Matlab/Simmechanics, it is translated into a base-mass-joint plot. By adding a senor and actuator in its library, the simulation of the output can be done. In our case, the mass-joint plot of the robot arm is in figure 4 and the simulation results of employing position control are in figure 5, in which $K_{p}=-100, K_{d}=-10$. The desired position is same as example1. 


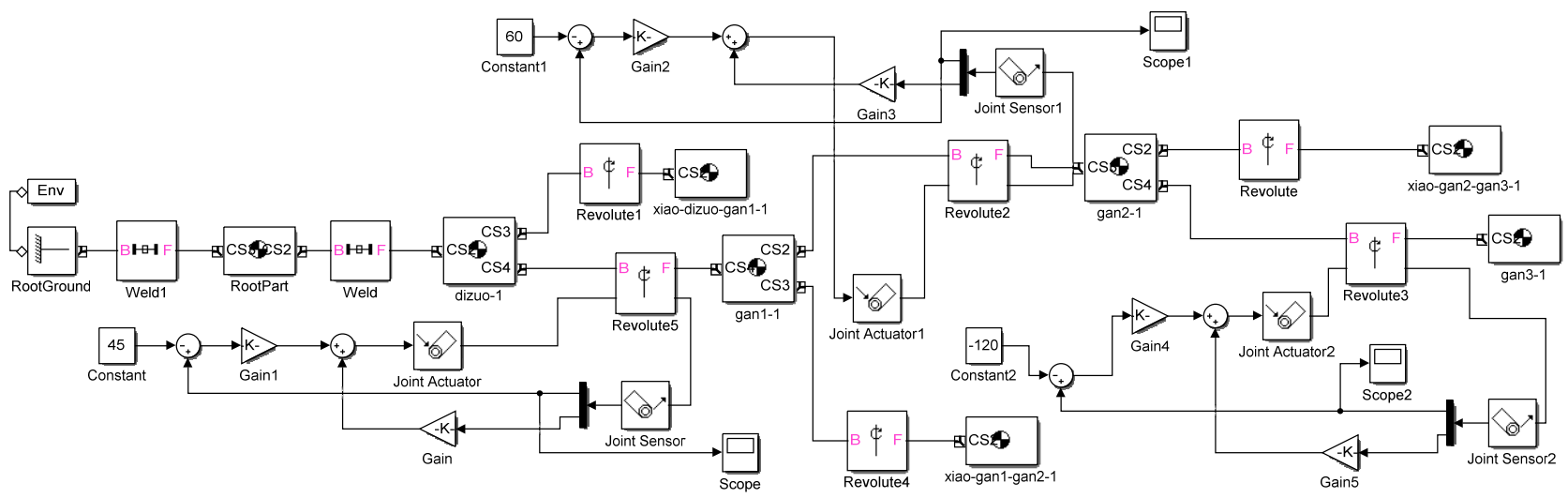

Figure. 4 the $3 \mathrm{~d}$ arm of the simulation layout in MATLAB SimMechanics
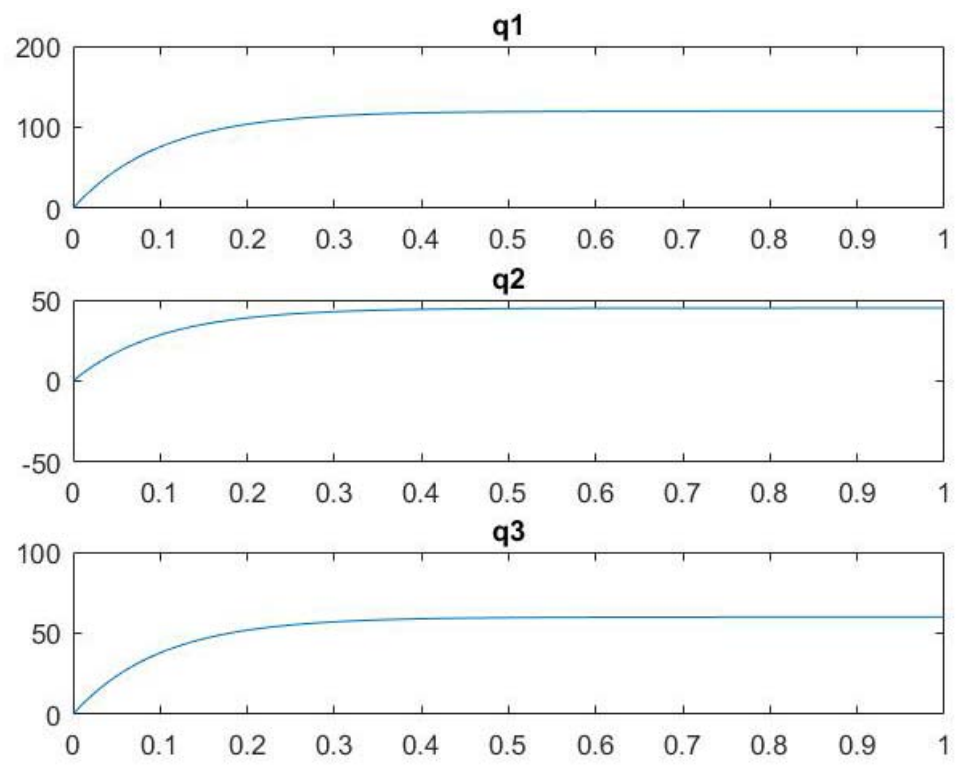

Figure.5 the position control in Simmechanic plot

\section{Conclusion}

In this paper, the modeling of a 3D arm from two approaches is presented. In nonlinear, a parameter-dependent system is obtained. Due to an existing inverse inertia, it is important to prove it is nonsingular such that the motion of the arm without imposing any limitation to avoid singularity. In linear, it is intuitive to build a model from SOLIDWORKS engineering drawing. Through Mtalab/Simmechanics translating, a base-mass-joint block diagram is obtained. It is advantageous for engineer to install some actuator input and sensor in Simmechanics library to predict the motion of the body. By implementing a position control law, both of them can reach the same desired position.

\section{References}

[1] Saeed B. Niku, Introduction to Robotics Analysis, Systems, Applications, Prentice Hall, New Jersey, 2001.

[2] I. Hassanzadeh, A. Harifi and F. Arvani, Design and Implementation of an Adaptive Control for a Robot, Am.J. of Appl. Sc. 4(2), pp. 56-59, 2007.

[3] C. Chen, M.M. and C.R. Bidlack, Simulation and animation of sensor-driven robots, IEEE Trans. On Robotics and Automation, 10(5), pp. 684-704, 1994. 
[4] M.J. Tsai, J.J. Fang and J.L. Chang, Robotic path planning for an automatic mold polishing system, International Journal of Robotic \& Automation, 19(2), pp. 81-89, 2004.

[5] Mark W. Spong, Seth Hutchinson, M. Vidyasagar, Robot Modeling and Control, John Wiley \& Sons, Inc. 2006.

[6] J. J Slotine, Weiping Li, Applied Nonlinear Control, Prentice Hall, New Jersey, 1991.

[7] Harry G. Kwatny, Gilmer L. Blankship, Nonlinear Control and Analytical Mechanics, Birkhauser, Boston, 2000.

[8] SIMULINK User's Guide, The Mathworks, Inc., 2007.

[9] M. Schlotter, Multibody System Simulation with SimMechanics, Technical Report, Darmstadt University of Technology, 2003.

[10] Arun Dayal Udai, C.G Rajeevlochana, Subir Kumar Saha, Dymamic Simulation of a KUKA KR5 Industrial Robot using MATLAB SimMechanics, $15^{\text {th }}$ National Conference on Machines and Mechanisms, 2011. 